\title{
Asymptotic Properties of Minimal Integration Rules
}

\author{
By Philip Rabinowitz and Nira Richter
}

\begin{abstract}
The error of a particular integration rule applied to a Hilbert space of functions analytic within an ellipse containing the interval of integration is a bounded linear functional. Its norm, which depends on the size of the ellipse, has proved useful in estimating the truncation error occurring when the integral of a particular analytic function is approximated using the rule in question. It is thus of interest to study rules which minimize this norm, namely minimal integration rules. The present paper deals with asymptotic properties of such minimal integration rules as the underlying ellipses shrink to the interval of integration.
\end{abstract}

1. Introduction. In the numerical integration of analytic functions, it is possible to define nonclassical integration rules, called Minimal Integration Rules, having a minimizing property relative to a subclass of analytic functions. To define these rules, we introduce Hilbert spaces of functions, analytic in simply connected domains $B$ in the complex plane, which contain the interval of integration $I$. The error in any integration rule, with abscissae in $I$, defines a bounded linear functional in each such Hilbert space. A rule for which the corresponding error functional is of minimum norm, relative to a particular Hilbert space, is called a minimal rule.

For the standardized interval $[-1,1]$, various minimal rules have been computed. In [8] Valentin dealt with Hilbert spaces of functions, analytic in circles with radius $R>1$. He proved that, as $R \rightarrow \infty$, the rules tend to Gaussian rules with the same number of points. A similar asymptotic behaviour was found by Barnhill [1], considering Hilbert spaces of analytic functions inside ellipses $\epsilon_{\rho}$, with foci at $( \pm 1,0)$ and semimajor axis $a=\frac{1}{2}(\sqrt{ } \rho+1 / \sqrt{ } \rho)$. In this case the minimal rules tend to Gaussian rules, as the areas of the ellipses tend to cover the complex plane $(\rho \rightarrow \infty)$.

The proofs given in [1] deal with spaces with an area integral as a scalar product. Similar proofs can be carried over for the case of a line integral scalar product [5].

In this work we discuss the asymptotic behaviour of minimal rules, as the ellipses collapse to the interval $I(\rho \rightarrow 1)$. For the two types of scalar products, the considerations are along the same lines, but the asymptotic behaviour turns out to be different. Using the same technique, we deal as well with minimal rules in the class of rules which integrate constants exactly.

The asymptotic behaviour of the minimal rules was checked numerically by computing minimal rules for various values of $\rho$, monotonically decreasing to 1 . The numerical results agree with the theoretical ones, as demonstrated in Section 7.

In Section 2 we introduce the two families of Hilbert spaces, $L^{2}\left(\epsilon_{\rho}\right), H^{2}\left(\epsilon_{\rho}\right)$, and formulate the two problems we deal with.

In Section 3 we cite a result which characterizes the minimizing weights in the

Received October 15, 1969.

AMS Subject Classifications. Primary 6555.

Key Words and Phrases. Hilbert space of analytic functions, norm of error functional, minimizing abscissae and weights, minimal norm integration rule, complete orthonormal set, asymptotic properties of minimal rules. 
general case, and prove a theorem, concerning the minimizing weights in the class of rules which integrate constants exactly.

In Section 4 we deal with the asymptotic behaviour of minimal rules, in the oneparameter family of spaces $L^{2}\left(\epsilon_{\rho}\right)$, as $\rho \rightarrow 1$. In Section 5 we give the analogous results in the family of spaces $H^{2}\left(\epsilon_{\rho}\right)$. In Section 6 we give a table which summarizes the results of Sections 4, 5 .

In Appendices A, B, we investigate the asymptotic behaviour, for $\rho \rightarrow 1$, of some infinite sums, the results being used in Sections 4, 5.

2. Formulation of the Problems. Let $\epsilon_{\rho}$ designate the ellipse with foci at $( \pm 1,0)$, where $\rho=(a+b)^{2}, a$ is the semimajor axis and $b$ is the semiminor axis, $b=\left(a^{2}-1\right)^{1 / 8}$. Two Hilbert spaces of analytic functions inside $\epsilon_{\rho}$ are considered:

(a) The collection of all analytic functions which satisfy:

$$
\iint_{e_{p}}|f(z)|^{2} d x d y<\infty .
$$

This collection [2, p. 207] constitutes a Hilbert space, $L^{2}\left(\epsilon_{\rho}\right)$, with a scalar product defined by:

$$
(f, g)=\iint_{\epsilon_{p}} f(z) \overline{g(z)} d x d y .
$$

A complete orthonormal set of functions in $L^{2}\left(\epsilon_{\rho}\right)$ can be expressed by the Chebyshev polynomials of the second kind [2, p. 241]:

$$
P_{n}(z)=\left(4(n+1) / \pi\left(\rho^{n+1}-\rho^{-n-1}\right)\right)^{1 / 2} U_{n}(z),
$$

where $U_{n}(z)=\sin (n+1) \theta / \sin \theta, \theta=\operatorname{arc} \cos z$.

(b) The collection of analytic functions which satisfy

$$
\int_{\partial \epsilon_{\rho}}|f(z)|^{2}\left|1-z^{2}\right|^{-1 / 2}|d z|<\infty
$$

constitutes a Hilbert space, $H^{2}\left(\epsilon_{\rho}\right)$, with a line integral scalar product

$$
(f, g)=\int_{\partial \epsilon_{\rho}} f(z) \overline{g(z)}\left|1-z^{2}\right|^{-1 / 2}|d z| .
$$

A complete orthonormal set of functions in $H^{2}\left(\epsilon_{\rho}\right)$ can be defined by the Chebyshev polynomials of the first kind [2, p. 240]:

$$
\begin{aligned}
& P_{n}(z)=\left(2 / \pi\left(\rho^{n}+\rho^{-n}\right)\right)^{1 / 2} T_{n}(z), \quad n \geqq 1, \\
& P_{0}(z)=1 /(2 \pi)^{1 / 2},
\end{aligned}
$$

where $T_{n}(z)=\cos n \theta, \theta=\operatorname{arc} \cos z$.

Any bounded linear functional $L$, in a Hilbert space $H$, determines by Riesz's representation theorem, a unique element $l \in H$, called the representer of $L$, which satisfies:

$$
L(f)=(f, l), \quad f \in H, \quad\|L\|=\|l\| .
$$


The error in a given numerical integration rule, with abscissae within the interval of integration $[-1,1]$, is a bounded linear functional, $E$, in both $L^{2}\left(\epsilon_{\rho}\right)$ and $H^{2}\left(\epsilon_{\rho}\right)$ (referred to as $H_{\rho}$ ). Hence:

$$
E(f)=\int_{-1}^{1} f(x) d x-\sum_{i=1}^{n} A_{i} f\left(x_{i}\right)=(f, r), \quad f \in H_{\rho},
$$

where $\left\{x_{i}, A_{i}\right\}_{i-1}^{n}$ are the abscissae and weights of the integration rule, and $r(z)$ is the representer of $E$. Now:

$$
r(z)=\phi(z)-\sum_{i=1}^{n} \bar{A}_{i} \phi_{x_{i}}(z),
$$

where $\phi(z), \phi_{x_{i}}(z), i=1, \cdots, n$, are the representers of the following functionals:

$$
\begin{array}{rlrl}
\int_{-1}^{1} f(x) d x & =(f, \phi), & f \in H_{\rho}, & \\
f\left(x_{i}\right) & =\left(f, \phi_{x_{i}}\right), & f \in H_{\rho}, \quad i=1, \cdots, n .
\end{array}
$$

Using the complete orthonormal set of functions in $H$, analytic expressions for the above representers can be derived:

$$
\begin{aligned}
\phi(z) & =\sum_{m=0}^{\infty} \overline{\left[\int_{-1}^{1} P_{m}(x) d x\right]} P_{m}(z), \\
\phi_{x i}(z) & =\sum_{m=0}^{\infty} \overline{P_{m}\left(x_{i}\right)} P_{m}(z), \quad i=1, \cdots, n .
\end{aligned}
$$

For a fixed set of $n$ distinct points in $[-1,1]$, two problems can be formulated:

Problem a. Determine the weights which minimize the norm on the error functional of the corresponding integration rule.

Problem b. Determine the weights which minimize the norm of the error functional, in the class of rules which integrate constants exactly. This restriction is equivalent to the side condition $\sum_{i=1}^{n} A_{i}=2$.

Having the minimizing weights as functions of the $n$ abscissae, it is possible to look for that set of $n$ abscissae, for which the corresponding error functional is of minimum norm, relative to any other set of $n$ abscissae in $[-1,1]$. This set will be referred to as the set of minimizing abscissae, and will lie in $(-1,1)[6]$.

In what follows, we consider, for both problems, the asymptotic behaviour, as $\rho \rightarrow 1$, of the minimizing weights, as functions of the abscissae, and the asymptotic behaviour of the minimizing abscissae as $\rho \rightarrow 1$.

3. Some Properties of the Minimizing Weights. The minimizing weights, in Problem a, satisfy the system of normal equations [2, p. 175]:

$$
\left(\phi, \phi_{x_{i}}\right)=\sum_{i=1}^{n} \overline{A_{j}}\left(\phi_{x_{i}}, \phi_{x_{i}}\right), \quad i=1, \cdots, n .
$$

System (5) possesses a unique solution whenever $\phi_{x_{1}}(z), \cdots, \phi_{x_{0}}(z)$ are linearly independent, which is the case when $x_{1}, \cdots, x_{n}$ are distinct. 
The norm of the error functional, with the set of minimizing weights, is given by:

$$
(r, r)=(\phi, \phi)-\sum_{i, j=1}^{n} A_{i} \overline{A_{j}}\left(\phi_{x_{i}}, \phi_{x i}\right)
$$

For the set of minimizing weights in Problem b (M.W.b), the following theorem is proved:

THEOREM 1. Given a set of $n$ distinct points in $(-1,1), x_{1}, \cdots, x_{n}$, the set of weights which solve the problem:

$$
\underset{A_{1} \ldots \ldots A_{n}}{\min }\left\|\phi-\sum_{i=1}^{n} \overline{A_{i}} \phi_{x_{i}}\right\|
$$

subject to the side condition $\sum_{i=1}^{n} A_{i}=2$, is unique. This set satisfies the following linear system of equations:

$$
\left(\phi, \phi_{x_{i}}\right)-\sum_{i=1}^{n} \overline{A_{i}}\left(\phi_{x_{i}}, \phi_{x_{i}}\right)=\mu, \quad i=1, \cdots, n,
$$

where $\mu$ is determined by the side condition.

Proof. Let

$$
r=\phi-\sum_{i=1}^{n} \overline{A_{i}} \phi_{x_{i}}=\phi-2 \phi_{x_{i}}-\sum_{i=2}^{n} \overline{A_{i}}\left(\phi_{x_{i}}-\phi_{x_{1}}\right) .
$$

The solution to the problem $\min _{A_{2}, \cdots, A_{n}}\|\boldsymbol{r}\|$ is given by the normal equations:

$$
\left(\phi-2 \phi_{x_{1}}, \phi_{x_{i}}-\phi_{x_{1}}\right)=\sum_{i=2}^{n} \overline{A_{j}}\left(\phi_{x_{j}}-\phi_{x_{1}}, \phi_{x_{i}}-\phi_{x_{2}}\right), \quad i=2, \cdots, n .
$$

Since the set of $n-1$ functions $\left\{\phi_{x_{i}}-\phi_{x_{1}}\right\}_{i=2}^{n}$ is linearly independent, $A_{2}, \cdots, A_{n}$ are uniquely determined, and with $A_{1}=2-\sum_{i=2}^{n} A_{i}$, the set of M.W.b is unique.

Rearranging the terms in (8) we get:

$$
\begin{aligned}
& \left(\phi, \phi_{x_{i}}\right)-\sum_{j=2}^{n} \overline{A_{i}}\left(\phi_{x_{i}}, \phi_{x_{i}}\right) \\
& =\left(2-\sum_{i=2}^{n} \overline{A_{i}}\right)\left(\phi_{x_{1}}, \phi_{x_{i}}\right)+\left(\phi, \phi_{x_{1}}\right)-\sum_{j=2}^{n} \overline{A_{i}}\left(\phi_{x_{i}}, \phi_{x_{1}}\right)-\left(2-\sum_{i=2}^{n} \overline{A_{i}}\right)\left(\phi_{x_{1}}, \phi_{x_{1}}\right), \\
& i=2, \cdots, n .
\end{aligned}
$$

Substitution of $A_{1}$ for $2-\sum_{i=2}^{n} A_{i}$, yields the desired result, namely:

$$
\left(\phi, \phi_{x_{i}}\right)-\sum_{i=1}^{n} \overline{A_{i}}\left(\phi_{x_{i}}, \phi_{x_{i}}\right)=\left(\phi, \phi_{x_{1}}\right)-\sum_{i=1}^{n} \overline{A_{i}}\left(\phi_{x_{i}}, \phi_{x_{1}}\right), \quad i=2, \cdots, n .
$$

COROllary 1. The vector of weights $\mathbf{A}=\left(A_{1}, \cdots, A_{n}\right)$, which satisfies (7), is given by: $\mathbf{A}=\mathrm{A}_{p}-v$ where $\mathrm{A}_{p}$ is the solution of $(5)$, and $v=\left(\nu_{1}, \cdots, \nu_{n}\right)$ is determined by the system:

$$
\sum_{i=1}^{n} \bar{\nu}_{i}\left(\phi_{x_{i}}, \phi_{x_{i}}\right)=\mu, \quad i=1, \cdots, n
$$


4. The Family of Spaces $L^{2}\left(\epsilon_{\rho}\right), \rho>1$. Explicit expressions for the representers $\phi(z), \phi_{x_{i}}(z)$ in $L^{2}\left(\epsilon_{\rho}\right)$, can be written, according to (1), (3), (4), in the following way:

$$
\begin{aligned}
\phi(z) & =(8 / \pi) \sum_{m=0}^{\infty}\left(\rho^{2 m+1}-\rho^{-2 m-1}\right)^{-1} U_{2 m}(z), \\
\phi_{x_{i}}(z) & =(4 / \pi) \sum_{m=0}^{\infty}(m+1)\left(\rho^{m+1}-\rho^{-m-1}\right)^{-1} U_{m}\left(x_{i}\right) U_{m}(z) .
\end{aligned}
$$

In (9) we used the known result: $\int_{-1}^{1} U_{m}(x) d x=\left[1+(-1)^{m}\right] /(m+1)$. Let $x_{1}, \cdots, x_{n}$ be any set of $n$ distinct points in $(-1,1)$. For $\rho>1$ the normal equations (5) multiplied by $(\rho-1 / \rho)^{2}$ will yield the minimizing weights:

$$
\sum_{i=1}^{n} \overline{A_{i}}(\rho-1 / \rho)^{2}\left(\phi_{x_{i}}, \phi_{x_{i}}\right)=(\rho-1 / \rho)^{2}\left(\phi, \phi_{x_{i}}\right), \quad j=1, \cdots, n .
$$

Now:

$$
(\rho-1 / \rho)^{2}\left(\phi, \phi_{x_{i}}\right)=(\rho-1 / \rho)^{2}(8 / \pi) \sum_{m=0}^{\infty}\left(\rho^{2 m+1}-\rho^{-2 m-1}\right)^{-1} U_{2 m}\left(x_{i}\right)
$$

and

$(\rho-1 / \rho)^{2}\left(\phi_{x_{i}}, \phi_{x_{j}}\right)=(\rho-1 / \rho)^{2}(4 / \pi) \sum_{m=0}^{\infty}(m+1)\left(\rho^{m+1}-\rho^{-m-1}\right)^{-1} U_{m}\left(x_{i}\right) U_{m}\left(x_{i}\right)$.

Since all the coefficients of system (11) are real, the solution of (11) is also real. Using the results of Appendix A, the above expressions, as $\rho \rightarrow 1$, are asymptotically:

$$
\begin{aligned}
(\rho-1 / \rho)^{2}\left(\phi, \phi_{x_{i}}\right) & =\left[2 /\left(1-x_{i}^{2}\right)^{1 / 2}+o(1)\right](\rho-1 / \rho), \\
(\rho-1 / \rho)^{2}\left(\phi_{x_{i}}, \phi_{x_{i}}\right) & =\left[\pi /\left(1-x_{i}^{2}\right)\right] \delta_{i j}+o(1) .
\end{aligned}
$$

Inserting the expressions in (11), we get:

$$
\sum_{i=1}^{n}\left[\pi \delta_{i i} /\left(1-x_{i}^{2}\right)+o(1)\right] A_{i}=(\rho-1 / \rho)\left[2 /\left(1-x_{i}^{2}\right)^{1 / 2}+o(1)\right], \quad j=1, \cdots, n .
$$

To first order in $(\rho-1 / \rho)$ the solution of the above system is the following:

$$
A_{i}=\left[2\left(1-x_{i}^{2}\right)^{1 / 2} / \pi+o(1)\right](\rho-1 / \rho), \quad i=1, \cdots, n .
$$

For this set of weights, $(r, r)$ is given by (6). Inserting (12) into (6), we get for $\rho \rightarrow 1$ :

$$
\begin{aligned}
(r, r) & =(\phi, \phi)-\sum_{i, j=1}^{n}\left[4\left(\left(1-x_{i}^{2}\right)\left(1-x_{i}^{2}\right)\right)^{1 / 2} / \pi^{2}+o(1)\right]\left[\pi \delta_{i j} /\left(1-x_{i}^{2}\right)+o(1)\right] \\
& =(\phi, \phi)-\sum_{i=1}^{n} 4 / \pi+o(1)=(\phi, \phi)-4 n / \pi+o(1),
\end{aligned}
$$

where

$$
\begin{aligned}
(\phi, \phi) & =\int_{-1}^{1} \phi(x) d x=2(\rho-1 / \rho)^{-1}\left[\int_{-1}^{1} d x /\left(1-x^{2}\right)^{1 / 2}+o(1)\right] \\
& =[2 \pi+o(1)](\rho-1 / \rho)^{-1}
\end{aligned}
$$


These results yield the following theorem:

THEOREM 2. For any set of $n$ distinct points $x_{1}, \cdots, x_{n}$ in $(-1,1)$, the minimizing weights converge to zero as $\rho \rightarrow 1$ according to $(13)$. Each point in $(-1,1)$ contributes asymptotically the same amount to the reduction of $(r, r)$, this amount being asymptotically $4 / \pi$. The ratio of this amount to the value of $(\phi, \phi)$ is $\left[2 / \pi^{2}+o(1)\right](\rho-1 / \rho)$, and $(r, r)$ is asymptotically equal to $(\phi, \phi)$, where

$$
(\phi, \phi)=[2 \pi+o(1)](\rho-1 / \rho)^{-1} .
$$

The minimizing weights of Problem b can be expressed, using (13) and Corollary 1, in the following way:

$$
A_{i}=\left[2\left(1-x_{i}^{2}\right)^{1 / 2} / \pi+o(1)\right](\rho-1 / \rho)-\nu_{i}, \quad i=1, \cdots, n,
$$

where $\nu_{1}, \cdots, \nu_{n}$ are the solution of the system:

$$
\sum_{i=1}^{n}\left[\pi \delta_{i j} /\left(1-x_{i}^{2}\right)+o(1)\right] \nu_{i}=\mu(\rho-1 / \rho)^{2}, \quad i=1, \cdots, n .
$$

$\mu$ is determined by the condition $\sum_{i=1}^{k} A_{i}=2$, namely:

$$
\left[2\left(1-x_{i}^{2}\right)^{1 / 2} / \pi+o(1)\right](\rho-1 / \rho)-2=\sum_{i=1}^{n} \nu_{i} .
$$

Now $\nu_{i}=\mu\left[\left(1-x_{i}^{2}\right) / \pi+o(1)\right](\rho-1 / \rho)^{2}$ and so we have:

$$
\mu=-\left[2 \pi / \sum_{i=1}^{n}\left(1-x_{i}^{2}\right)+o(1)\right](\rho-1 / \rho)^{-2}
$$

and

$$
A_{i}=2\left(1-x_{i}^{2}\right) / \sum_{i=1}^{n}\left(1-x_{i}^{2}\right)+o(1) .
$$

This result furnishes the first part of the following theorem:

THEOREM 3. For any set of $n$ distinct points in $(-1,1)$, the minimizing weights which satisfy $\sum_{i=1}^{n} A_{i}=2$, are asymptotically given by (14). The norm of the corresponding error functional behaves asymptotically as $\left(\pi / \sum_{i=1}^{n}\left(1-x_{i}^{2}\right)\right)^{1 / 2} 2(\rho-1 / \rho)^{-1}$, and the minimizing abscissae tend to the midpoint of $I$, namely to zero. The corresponding minimizing weights tend to be equal, each tending to $2 / n$.

Proof. The general form of $(r, r)$ is:

$$
(r, r)=(\phi, \phi)-2 \sum_{i=1}^{n} A_{i}\left(\phi, \phi_{x_{i}}\right)+\sum_{i, j=1}^{n} A_{i} A_{j}\left(\phi_{x_{i}}, \phi_{x_{i}}\right) .
$$

Inserting the asymptotic expressions, (12) for the scalar products, and (14) for the weights, we get after some manipulations:

$$
(r, r)=\left[4 \pi / \sum_{i=1}^{n}\left(1-x_{i}^{2}\right)+o(1)\right](\rho-1 / \rho)^{-2} .
$$

From this expression we conclude that the minimizing abscissae tend to maximize 
the sum $\sum_{i=1}^{n}\left(1-x_{i}^{2}\right)$, namely $x_{i} \rightarrow 0, i=1, \cdots, n$. By (14) the corresponding minimizing weights tend to be equal:

$$
A_{i}=2\left(1-x_{i}^{2}\right) / \sum_{i=1}^{n}\left(1-x_{i}^{2}\right)+o(1) \rightarrow 2 / n .
$$

5. The Family of Spaces $H^{2}\left(\epsilon_{p}\right), \rho>1$. The representers $\phi(z), \phi_{x_{0}}(z)$ in $H^{2}\left(\epsilon_{\rho}\right)$ are expressed, using (2), (3), (4), in the following way:

$$
\begin{aligned}
\phi(z) & =(4 / \pi) \sum_{m=0}^{\infty},\left[\left(1-4 m^{2}\right)\left(\rho^{2 m}+\rho^{-2 m}\right)\right]^{-1} T_{2 m}(z), \\
\phi_{x i}(z) & =(2 / \pi) \sum_{m=0}^{\infty}\left(\rho^{m}+\rho^{-m}\right)^{-1} T_{m}\left(x_{i}\right) T_{m}(z),
\end{aligned}
$$

where the prime indicates that the term for $m=0$ is to be halved. In (16) we used the known result:

$$
\begin{aligned}
\int_{-1}^{1} T_{m}(x) d x & =\left[1+(-1)^{m}\right] /\left(1-m^{2}\right), & & m \neq 1, \\
& =0, & & m=1 .
\end{aligned}
$$

Let $x_{1}, \cdots, x_{n}$ be a set of $n$ distinct points in $(-1,1)$. For $\rho>1$ the normal equations (5) multiplied by $(\rho-1 / \rho)$ yield the minimizing weights:

$$
\sum_{i=1}^{n} \overline{A_{i}}(\rho-1 / \rho)\left(\phi_{x_{i}}, \phi_{x_{i}}\right)=(\rho-1 / \rho)\left(\phi, \phi_{x_{i}}\right), \quad j=1, \cdots, n .
$$

Now:

$$
(\rho-1 / \rho)\left(\phi_{x_{i}}, \phi_{x_{i}}\right)=(\rho-1 / \rho)(2 / \pi) \sum_{m=0}^{\infty}\left(\rho^{m}+\rho^{-m}\right)^{-1} T_{m}\left(x_{i}\right) T_{m}\left(x_{i}\right),
$$

and

$$
(\rho-1 / \rho)\left(\phi, \phi_{x_{i}}\right)=(\rho-1 / \rho)(4 / \pi) \sum_{m=0}^{\infty},\left[\left(\rho^{2 m}+\rho^{-2 m}\right)\left(1-4 m^{2}\right)\right]^{-1} T_{2 m}\left(x_{i}\right) .
$$

Since all the coefficients of system (18) are real, the solution of (18) is real as well. Applying the results of Appendix B, the above expressions, as $\rho \rightarrow 1$, are asymptotically:

$$
\begin{aligned}
(\rho-1 / \rho)\left(\phi_{x_{i}}, \phi_{x_{i}}\right) & =\frac{1}{2} \delta_{i j}+o(1) \\
(\rho-1 / \rho)\left(\phi, \phi_{x_{i}}\right) & =\left[\frac{1}{2}\left(1-x_{i}^{2}\right)^{1 / 2}+o(1)\right](\rho-1 / \rho) .
\end{aligned}
$$

Inserting these expressions in (18), we get:

$$
\sum_{i=1}^{n}\left[\frac{1}{2} \delta_{i j}+o(1)\right] A_{i}=(\rho-1 / \rho)\left[\frac{1}{2}\left(1-x_{i}^{2}\right)^{1 / 2}+o(1)\right], \quad j=1, \cdots, n .
$$

The solution of $(20)$ to first order in $(\rho-1 / \rho)$ is:

$$
A_{i}=\left[\left(1-x_{i}^{2}\right)^{1 / 2}+o(1)\right](\rho-1 / \rho), \quad i=1, \cdots, n .
$$


For these weights, $(r, r)$ can be written by (6) and (19) in the following way:

$$
\begin{aligned}
(r, r) & =(\phi, \phi)-(\rho-1 / \rho) \sum_{i, j=1}^{n}\left[\left(\left(1-x_{i}^{2}\right)\left(1-x_{j}^{2}\right)\right)^{1 / 2}+o(1)\right]\left[\frac{1}{2} \delta_{i j}+o(1)\right] \\
\quad & =(\phi, \phi)-(\rho-1 / \rho)\left[\frac{1}{2} \sum_{i=1}^{n}\left(1-x_{i}^{2}\right)+o(1)\right],
\end{aligned}
$$

where

$$
(\phi, \phi)=\int_{-1}^{1} \phi(x) d x=\frac{1}{2} \int_{-1}^{1}\left(1-x^{2}\right)^{1 / 2} d x+o(1)=\pi / 4+o(1) .
$$

Thus the minimizing abscissae tend to maximize $\sum_{i=1}^{n}\left(1-x_{i}^{2}\right)$, so that $x_{i} \rightarrow 0$, $i=1, \cdots, n$.

These results are formulated in the following theorem:

THEOREM 4. For any set of $n$ distinct points $x_{1}, \cdots, x_{n}$ in $(-1,1)$, the minimizing weights tend to zero as $\rho \rightarrow 1$, according to (21). The norm of the error functional, for this set of weights tends to $\|\phi\|=\sqrt{ } \pi / 2$, and the amount by which any point $x_{i}$, reduces $(r, r)$ is asymptotically $\frac{1}{2}\left(1-x_{i}^{2}\right)(\rho-1 / \rho)$. The minimizing abscissae tend to zero, as $\rho \rightarrow 1$, and the corresponding minimizing weights turn out to be equal.

The minimizing weights of Problem b, by (21) and Corollary 1, are:

$$
A_{i}=\left[\left(1-x_{i}^{2}\right)^{1 / 2}+o(1)\right](\rho-1 / \rho)-\nu_{i}, \quad i=1, \cdots, n,
$$

where $\nu_{1}, \cdots, \nu_{n}$ are the solution of the system:

$$
\sum_{i=1}^{n}\left[\frac{1}{2} \delta_{i j}+o(1)\right](\rho-1 / \rho) \nu_{i}=\mu, \quad i=1, \cdots, n,
$$

and $\mu$ is determined by the condition:

$$
-\sum_{i=1}^{n} \nu_{i}=2-(\rho-1 / \rho)\left[\sum_{i=1}^{n}\left(1-x_{i}^{2}\right)^{1 / 2}+o(1)\right] .
$$

Now:

$$
\begin{aligned}
\nu_{i} & =[2 \mu+o(1)](\rho-1 / \rho), \\
\mu & =[-1 / n+o(1)](\rho-1 / \rho)^{-1}
\end{aligned}
$$

and

$$
A_{i}=2 / n+o(1), \quad i=1, \cdots, n .
$$

Inserting this result in (15) and using (19), we get after some manipulations:

$$
(r, r)=[2 / n+o(1)](\rho-1 / \rho)^{-1} .
$$

These results are summarized in the following theorem:

THEOREM 5. For any set of $n$ distinct points in $(-1,1)$, the minimizing weights, in 
the class of weights which satisfy $\sum_{i=1}^{n} A_{i}=2$, are asymptotically equal, each tending to $2 / n$ as $\rho \rightarrow 1$. The norm of the corresponding error functional increases as $(2 / n)^{1 / 2}(\rho-1 / \rho)^{-1 / 2}$. Each point in $(-1,1)$ is "equally good", with respect to the asymptotic contribution to the reduction of $(r, r)$.

6. Summary of Results.

\begin{tabular}{lll}
\hline & \multicolumn{1}{c}{$L^{2}\left(\epsilon_{\rho}\right)$} & \multicolumn{1}{c}{$H^{2}\left(\epsilon_{\rho}\right)$} \\
\hline \multicolumn{1}{c}{ Minimal Rules } \\
\hline $\begin{array}{l}\text { Minimizing } \\
\text { Weights } \\
\text { for Fixed }\end{array}$ & $A_{i} \sim(2 / \pi)\left(1-x_{i}^{2}\right)^{1 / 2}(\rho-1 / \rho)$ & $A_{i} \sim\left(1-x_{i}^{2}\right)^{1 / 2}(\rho-1 / \rho)$ \\
Abscissae & $\|r\| \sim 2 \pi(\rho-1 / \rho)^{-1 / 2}$ & $\|r\| \sim \pi / 4$ \\
\hline Rules with & $x_{i} \sim$ not known & \\
Minimizing & $A_{i} \sim(2 / \pi)\left(1-x_{i}^{2}\right)^{1 / 2}(\rho-1 / \rho)$ & $x_{i} \sim 0$ \\
Abscissae & $\|r\| \sim(2 \pi /(\rho-1 / \rho))^{1 / 2}$ & $A_{i} \sim(\rho-1 / \rho)$ \\
\hline
\end{tabular}

\begin{tabular}{cc}
\hline$L^{2}\left(\epsilon_{p}\right)$ & $H^{2}\left(\epsilon_{\rho}\right)$ \\
\hline
\end{tabular}

Minimal Rules with $\sum_{i=1}^{n} A_{i}=2$

\begin{tabular}{|c|c|c|}
\hline $\begin{array}{l}\text { Minimizing } \\
\text { Weights } \\
\text { for Fixed } \\
\text { Abscissae }\end{array}$ & $\begin{array}{c}A_{i} \sim 2\left(1-x_{i}^{2}\right) / \sum_{i=1}^{n}\left(1-x_{i}^{2}\right) \\
\|r\| \sim 2\left(\pi / \sum_{i=1}^{n}\left(1-x_{i}^{2}\right)\right)^{1 / 2}(\rho-1 / \rho)^{-1}\end{array}$ & $\|r\| \sim(2 / n)^{1 / 2}(\rho-1 / \rho)^{-1 / 2}$ \\
\hline $\begin{array}{l}\text { Rules with } \\
\text { Minimizing } \\
\text { Abscissae }\end{array}$ & $\begin{array}{l}x_{i} \sim 0 \\
A_{i} \sim 2 / n \\
\|r\| \sim 2(\pi / n)^{1 / 2}(\rho-1 / \rho)^{-1}\end{array}$ & $\begin{array}{l}x_{i} \sim \text { not known } \\
A_{i} \sim 2 / n \\
\|r\| \sim(2 / n)^{1 / 2}(\rho-1 / \rho)^{-1 / 2}\end{array}$ \\
\hline
\end{tabular}

7. Tables. The following four tables demonstrate the asymptotic behaviour, as $\rho \rightarrow 1$, of minimal rules with 2, 3, 4 abscissae.

Since all the rules turned out to be symmetric, the given abscissae are all nonnegative.

In Table 1 the rules are unconstrained minimal rules in $L^{2}\left(\epsilon_{\rho}\right)$, while in Table 2 the rules are minimal rules with $\sum_{i=1}^{n} A_{i}=2$. Tables 3,4 are the analogous tables for $H^{2}\left(\epsilon_{\rho}\right)$.

Remark. In Table 1 the minimizing abscissae are inaccurate, since any point in $(-1,1)$ is "equally good" in this case, and the function $(r, r)$ is very "flat". However, by inspection, we conjecture that the minimizing abscissae converge, as $\rho \rightarrow 1$, to the roots of $U_{n}(x)$, namely: $x_{i} \rightarrow \cos i \pi /(n+1), i=1, \cdots, n$. This behaviour cannot be proved by our methods, since it depends on higher orders of $(\rho-1 / \rho)$. 
TABLE 1

Minimal Rules in $L^{2}\left(\epsilon_{\rho}\right)$

\begin{tabular}{|c|c|c|c|c|c|c|c|c|c|c|}
\hline \multirow{3}{*}{$a$} & \multicolumn{10}{|c|}{$n$} \\
\hline & \multicolumn{2}{|c|}{2} & \multicolumn{4}{|c|}{3} & \multicolumn{4}{|c|}{4} \\
\hline & $x_{1}$ & $A_{1}$ & $x_{1}$ & $A_{1}$ & $x_{2}$ & $A_{2}$ & $x_{1}$ & $A_{1}$ & $x_{2}$ & $A_{2}$ \\
\hline 1.01 & .5179 & .3078 & .7288 & .2462 & 0.0 & .3596 & .8303 & .1994 & .3208 & .3389 \\
\hline 1.005 & .5127 & .2185 & .7225 & .1760 & 0.0 & .2546 & .8243 & .1441 & .3174 & .2413 \\
\hline 1.001 & .4972 & .0988 & .7180 & .0793 & 0.0 & .1138 & .8271 & .0640 & .3420 & .1070 \\
\hline 1.0005 & .4954 & .0699 & .6940 & .0580 & 0.0 & .0806 & - & - & - & - \\
\hline 1.0001 & .4936 & .0313 & .6916 & .0260 & 0.0 & .0360 & .8192 & .0207 & .3395 & .0339 \\
\hline
\end{tabular}

TABLE 2

Constrained Minimal Rules in $L^{2}\left(\epsilon_{\rho}\right) \cdot\left(\sum_{i=1}^{n} A_{i}=2\right)$

\begin{tabular}{|c|c|c|c|c|c|c|c|c|c|c|}
\hline \multirow{3}{*}{$a$} & \multicolumn{10}{|c|}{$n$} \\
\hline & \multicolumn{2}{|c|}{2} & \multicolumn{4}{|c|}{3} & \multicolumn{4}{|c|}{4} \\
\hline & $x_{1}$ & $A_{1}$ & $x_{1}$ & $A_{1}$ & $x_{2}$ & $A_{2}$ & $x_{1}$ & $A_{1}$ & $x_{2}$ & $A_{2}$ \\
\hline 1.01 & .2471 & 1.0 & .4485 & .6323 & 0.0 & .7354 & .6211 & .4311 & .2140 & .5689 \\
\hline 1.005 & .1911 & 1.0 & .3480 & .6457 & 0.0 & .7086 & .4849 & .4590 & .1625 & .5481 \\
\hline 1.001 & .1040 & 1.0 & .1914 & .6600 & 0.0 & .6800 & .2695 & .4869 & .0883 & .5131 \\
\hline 1.0005 & .0795 & 1.0 & .1470 & .6627 & 0.0 & .6746 & .2077 & .4921 & .0679 & .5079 \\
\hline 1.0001 & .0419 & 1.0 & .0782 & .6655 & 0.0 & .6690 & - & - & - & - \\
\hline
\end{tabular}

TABLE 3

Minimal Rules in $H^{2}\left(\epsilon_{\rho}\right)$

$n$

\begin{tabular}{|c|c|c|c|c|c|c|c|c|c|c|}
\hline \multirow[t]{2}{*}{$a$} & \multicolumn{2}{|c|}{2} & \multicolumn{4}{|c|}{3} & \multicolumn{4}{|c|}{4} \\
\hline & $x_{1}$ & $A_{1}$ & $x_{1}$ & $A_{1}$ & $x_{2}$ & $A_{2}$ & $x_{1}$ & $A_{1}$ & $x_{2}$ & $A_{2}$ \\
\hline 01 & .3240 & .6195 & .5266 & .4958 & 0.0 & .5374 & .6605 & .3977 & .2281 & .4560 \\
\hline 1.005 & .2698 & .4930 & .4525 & .4187 & 0.0 & .4418 & .5829 & .3547 & .1974 & .388 \\
\hline 1.001 & .1629 & .2586 & .2879 & .2403 & 0.0 & .2450 & .3895 & .2225 & .1282 & .230 \\
\hline 1.0005 & .1279 & .1898 & .2294 & .1803 & 0.0 & .1826 & .3148 & 1709 & .1030 & .1746 \\
\hline 1.0001 & .0703 & .0891 & 1292 & .0872 & 0.0 & .0876 & .1812 & .0853 & .0590 & .0859 \\
\hline
\end{tabular}


TABLE 4

Constrained Minimal Rules in $H^{2}\left(\epsilon_{\rho}\right) \cdot\left(\sum_{i=1}^{n} A_{i}=2\right)$

\begin{tabular}{|c|c|c|c|c|c|c|c|c|c|c|}
\hline \multirow{3}{*}{$a$} & \multicolumn{10}{|c|}{$n$} \\
\hline & \multicolumn{2}{|c|}{2} & \multicolumn{4}{|c|}{3} & \multicolumn{4}{|c|}{4} \\
\hline & $x_{1}$ & $A_{1}$ & $x_{1}$ & $A_{1}$ & $x_{2}$ & $A_{2}$ & $x_{1}$ & $A_{1}$ & $x_{2}$ & $A_{2}$ \\
\hline 1.01 & .3566 & 1.0 & .5572 & .6505 & 0.0 & 6990 & .6832 & .4678 & .2374 & .5322 \\
\hline 1.005 & .3054 & 1.0 & .4919 & .6570 & 0.0 & .6860 & .6169 & .4800 & .2107 & .5200 \\
\hline 1.001 & .1956 & 1.0 & .3342 & .6644 & 0.0 & .6712 & .4403 & . 4947 & .1463 & .5053 \\
\hline 1.0005 & .1569 & 1.0 & .2732 & .6655 & 0.0 & .6690 & .3659 & .4973 & 1209 & .5027 \\
\hline 1.0001 & .0898 & 1.0 & .1614 & .6665 & 0.0 & .6670 & .2223 & .4995 & .0730 & .5005 \\
\hline
\end{tabular}

In Table 4 we observe a slow convergence of the minimizing abscissae to zero, a behaviour which cannot be predicted by the theory.

The minimization of $(r, r)$ as a function of the abscissae and weights, was performed by the method of Fletcher and Powell [3].

\section{APPENDIX A}

The first two results are cited from [7, Chapter 1].

Result 1 (Abel). If $b_{1} \geqq b_{2} \geqq \cdots \geqq b_{n} \geqq \cdots \geqq 0$, and if $m \leqq a_{1}+a_{2}+\cdots+$ $a_{n} \leqq M$ for all values of $n$, then:

$$
b_{1} m \leqq a_{1} b_{1}+a_{2} b_{2}+\cdots+a_{n} b_{n} \leqq b_{1} M, \quad \text { for all values of } n \text {. }
$$

Result $2(\mathrm{Abel})$. Let $\sum_{n=0}^{\infty} a_{n} \psi_{n}(x)$ be uniformly convergent in any closed interval contained in $(\alpha, \beta)$. If $\sum_{n=0}^{\infty} a_{n}<\infty$, and if $0<\psi_{n}(x) \leqq 1$ for $x \in(\alpha, \beta)$, and $\psi_{n}(\alpha)=1$, then $\sum_{n=0}^{\infty} a_{n} \psi_{n}(x)$ is uniformly convergent in $\alpha \leqq x \leqq \beta_{1}<\beta$, and

$$
\lim _{x \rightarrow \alpha} \sum_{n=0}^{\infty} a_{n} \psi_{n}(x)=\sum_{n=0}^{\infty} a_{n} .
$$

In what follows $U_{n}(x)$ denotes the Chebyshev polynomial of the second kind, $U_{n}(x)=$ $\sin (n+1) \theta / \sin \theta$, with $\theta=\operatorname{arc} \cos x$.

LEMMA 1. For $-1<x<1$,

$$
\lim _{\rho \rightarrow 1^{+}}(\rho-1 / \rho) \sum_{m=0}^{\infty} 2\left(\rho^{2 m+1}-\rho^{-2 m-1}\right)^{-1} U_{2 m}(x)=\pi / 2\left(1-x^{2}\right)^{1 / 2} .
$$

Proof. Substitution of $\theta=\operatorname{arc} \cos x$ in the left-hand side of the above expression yields:

$$
\begin{aligned}
2(\rho-1 / \rho)(\sin \theta)^{-1} & \sum_{m=0}^{\infty}\left(\rho^{2 m+1}-\rho^{-2 m-1}\right)^{-1} \sin (2 m+1) \theta \\
= & \left(2 /\left(1-x^{2}\right)^{1 / 2}\right) \sum_{m=0}^{\infty}\left(\rho^{2 m}+\rho^{2 m-2}+\cdots+\rho^{-2 m}\right)^{-1} \sin (2 m+1) \theta \\
= & \left(2 /\left(1-x^{2}\right)^{1 / 2}\right) \sum_{m=0}^{\infty} b_{m}(\rho) a_{m}(\theta),
\end{aligned}
$$


where

$$
b_{m}(\rho)=(2 m+1) /\left(\rho^{2 m}+\cdots+\rho^{-2 m}\right)
$$

and

$$
a_{m}(\theta)=\sin (2 m+1) \theta /(2 m+1) .
$$

Now $0<b_{m}(\rho)<1$ for any $\rho>1$ and $b_{m}(1)=1$. By [4, p. 38]

$$
\sum_{m=0}^{\infty} a_{m}(\theta)=\pi / 4
$$

The sum $\sum_{m=0}^{\infty} b_{m}(\rho) a_{m}(\theta)$ is uniformly convergent for any $\rho \geqq \rho^{*}>1$. Applying Result 2 we get:

$$
\lim _{\rho \rightarrow 1} \sum_{m=0}^{\infty} b_{m}(\rho) a_{m}(\theta)=\sum_{m=0}^{\infty} a_{m}(\theta)=\pi / 4,
$$

and the desired result follows.

LemMa 2. For $-1<x_{i}, x_{i}<1, x_{i} \neq x_{i}$,

$$
\lim _{\rho \rightarrow 1^{+}}(\rho-1 / \rho)^{2} \sum_{m=0}^{\infty}(m+1)\left(\rho^{m+1}-\rho^{-m-1}\right)^{-1} U_{m}\left(x_{i}\right) U_{m}\left(x_{i}\right)=0 .
$$

Proof.

$$
\begin{aligned}
(\rho-1 / \rho) \sum_{m=0}^{\infty}(m+1)\left(\rho^{m+1}-\rho^{-m-1}\right)^{-1} U_{m}\left(x_{i}\right) U_{m}\left(x_{i}\right) \\
=\left[\left(1-x_{i}^{2}\right)\left(1-x_{i}^{2}\right)\right]^{-1 / 2} \sum_{m=0}^{\infty} b_{m}(\rho) a_{m}\left(\theta_{i}, \theta_{i}\right)
\end{aligned}
$$

where

$$
\begin{aligned}
b_{m}(\rho) & =(m+1) /\left(\rho^{m}+\cdots+\rho^{-m}\right), \\
a_{m}\left(\theta_{i}, \theta_{i}\right) & =\sin (m+1) \theta_{i} \sin (m+1) \theta_{i}
\end{aligned}
$$

with $\theta_{i}=\arccos x_{i}, \theta_{i}=\arccos x_{i}$, and $0<\theta_{i}, \theta_{i}<\pi$.

Now $a_{m}\left(\theta_{i}, \theta_{i}\right)=\frac{1}{2}[\cos (m+1) \alpha-\cos (m+1) \beta]$ where $\alpha=\left|\theta_{i}-\theta_{i}\right| \neq 0$, and $\beta=\theta_{i}+\theta_{i}<2 \pi$. For any $\rho>1,0<b_{m}(\rho)<1$ and $b_{m+1}(\rho)<b_{m}(\rho)$. This is true since with $\rho=e^{x}, x>0$,

$$
b_{m}=(m+1) \sinh x / \sinh (m+1) x
$$

and

$$
\frac{\partial b_{m}}{\partial m}=\sinh x \cosh (m+1) x[\tanh (m+1) x-(m+1) x] / \sinh ^{2}(m+1) x,
$$

but tanh $y-y<0$ for $y>0$, and thus $\partial b_{m} / \partial m<0$. Using the identity [4, p. 30]:

$$
\sum_{k=0}^{n} \cos k x=\cos \frac{n x}{2} \sin \frac{(n+1) x}{2} \operatorname{cosec} \frac{x}{2}
$$


we get

$$
\sum_{m=0}^{n-1} a_{m}=\frac{1}{2}\left\{\cos \frac{n \alpha}{2} \sin \frac{(n+1) \alpha}{2} \operatorname{cosec} \frac{\alpha}{2}-\cos \frac{n \beta}{2} \cdot \sin \frac{(n+1) \beta}{2} \operatorname{cosec} \frac{\beta}{2}\right\},
$$

and for any value of $N,-M \leqq \sum_{m=0}^{N} a_{m} \leqq M$, where $M=\frac{1}{2}[\operatorname{cosec} \alpha / 2+\operatorname{cosec} \beta / 2]$. Applying Result 1, we get:

$$
-M b_{0} \leqq \sum_{m=0}^{\infty} a_{m} b_{m} \leqq M b_{0}
$$

and since $b_{0}=1$, we have for $\rho>1$ :

$$
\begin{array}{r}
(\rho-1 / \rho)^{2}\left|\sum_{m=0}^{\infty}(m+1)\left(\rho^{m+1}-\rho^{-m-1}\right)^{-1} U_{m}\left(x_{i}\right) U_{m}\left(x_{i}\right)\right| \\
\leqq(\rho-1 / \rho) M /\left(\left(1-x_{i}^{2}\right)\left(1-x_{i}^{2}\right)\right)^{1 / 2}
\end{array}
$$

This relation yields the desired result when $\rho \rightarrow 1^{+}$.

LEMMA 3. For $0<\theta<\pi$,

$$
\begin{aligned}
& \lim _{\rho \rightarrow 1^{+}}\left\{(\rho-1 / \rho)^{2} \sum_{m=1}^{\infty} m\left(\rho^{m}-\rho^{-m}\right)^{-1} \sin ^{2} m \theta\right. \\
& \left.-(\rho-1 / \rho)^{2}(1 / 2) \sum_{m=1}^{\infty} m\left(\rho^{m}-\rho^{-m}\right)^{-1}\right\}=0 .
\end{aligned}
$$

Proof. For any $\rho>1$ we have:

$$
\begin{aligned}
2(\rho-1 / \rho) \sum_{m=1}^{\infty} & m\left(\rho^{m}-\rho^{-m}\right)^{-1} \sin ^{2} m \theta-(\rho-1 / \rho) \sum_{m=1}^{\infty} m\left(\rho^{m}-\rho^{-m}\right)^{-1} \\
& =(\rho-1 / \rho) \sum_{m=1}^{\infty} m\left(\rho^{m}-\rho^{-m}\right)^{-1}\left(2 \sin ^{2} m \theta-1\right)=\sum_{m=1}^{\infty} b_{m}(\rho) a_{m}(\theta),
\end{aligned}
$$

where $a_{m}(\theta)=2 \sin ^{2} m \theta-1$ and $b_{m}(\rho)=m(\rho-1 / \rho)\left(\rho^{m}-\rho^{-m}\right)^{-1}$. Applying the known result $[4$, p. 30$]$ :

$$
\sum_{m=1}^{N} \sin ^{2} m \theta=\frac{N}{2}-\frac{\cos (N+1) \theta \sin N \theta}{2 \sin \theta}, \quad 0<\theta<\pi,
$$

we get the relation: $-1 / \sin \theta \leqq \sum_{m-1}^{N} a_{m}(\theta) \leqq 1 /$ sin $\theta$. Since $0<b_{m+1}<b_{m}$, as proved in Lemma 2, it is possible to apply Result 1 and get for any value of $N$ the following inequality:

$$
\left|\sum_{m=0}^{N} a_{m} b_{m}\right| \leqq 1 / \sin \theta
$$

Hence for any $\rho>1$ :

$$
\left|(\rho-1 / \rho)^{2} \sum_{m=1}^{\infty}\left(\rho^{m}-\rho^{-m}\right)^{-1}\left(2 \sin ^{2} m \theta-1\right)\right| \leqq(\rho-1 / \rho) / \sin \theta
$$

and as $\rho \rightarrow 1^{+}$the above expression tends to zero. 
LeMMA 4.*

$$
\lim _{\rho \rightarrow 1^{+}}(\rho-1 / \rho)^{2} \sum_{m=1}^{\infty} m\left(\rho^{m}-\rho^{-m}\right)^{-1}=\pi^{2} / 2 .
$$

Proof: The substitution $\rho=e^{x}, x>0$ yields:

$$
\begin{aligned}
(\rho-1 / \rho)^{2} \sum_{m=1}^{\infty} m\left(\rho^{m}-\rho^{-m}\right)^{-1} & =2 \sinh ^{2} x \sum_{m=1}^{\infty} \frac{m}{\sinh m x} \\
& =\frac{2 \sinh ^{2} x}{x^{2}} \sum_{m=1}^{\infty} \frac{m x^{2}}{\sinh m x} .
\end{aligned}
$$

Now

$$
\frac{m x^{2}}{\sinh m x} \leqq \int_{(m-1) x}^{m x} \frac{y d y}{\sinh y} \leqq \frac{(m-1) x^{2}}{\sinh (m-1) x},
$$

since $y / \sinh y$ is monotonically decreasing for $y>0$. Hence

$$
\int_{x}^{\infty} \frac{y d y}{\sinh y} \leqq \sum_{m=1}^{\infty} \frac{m x^{2}}{\sinh m x} \leqq \int_{0}^{\infty} \frac{y d y}{\sinh y},
$$

and in the limit $x \rightarrow 0^{+}\left(\rho \rightarrow 1^{+}\right)$we get:

$$
\lim _{x \rightarrow 0^{+}} \frac{2 \sinh ^{2} x}{x^{2}} \sum_{m=1}^{\infty} \frac{m x^{2}}{\sinh m x}=2 \int_{0}^{\infty} \frac{y d y}{\sinh y}=\frac{\pi^{2}}{2} .
$$

An immediate consequence of Lemmas 3, 4 is:

Corollary 1. For $-1<x<1$,

$$
\lim _{\rho \rightarrow 1^{+}}(\rho-1 / \rho)^{2} \sum_{m=0}^{\infty}(m+1)\left(\rho^{m+1}-\rho^{-m-1}\right)^{-1} U_{m}^{2}(x)=\pi^{2} / 4\left(1-x^{2}\right) .
$$

A summary of the results, which are referred to in Section 4, is given below: For $\rho \rightarrow 1^{+}$and $-1<x_{i}, x_{i}<1$ :

$$
(\rho-1 / \rho) \sum_{m=0}^{\infty} 2\left(\rho^{2 m+1}-\rho^{-2 m-1}\right)^{-1} U_{2 m}\left(x_{i}\right)=\pi / 2\left(1-x_{i}^{2}\right)^{1 / 2}+o(1),
$$

(2) $(\rho-1 / \rho)^{2} \sum_{m=0}^{\infty}(m+1)\left(\rho^{m+1}-\rho^{-m-1}\right)^{-1} U_{m}\left(x_{i}\right) U_{m}\left(x_{i}\right)=\pi^{2} \delta_{i i} / 4\left(1-x_{i}^{2}\right)+o(1)$.

\section{APPENDIX B}

In what follows $T_{n}(x)$ denotes the Chebyshev polynomial of the first kind, $T_{n}(x)=$ $\cos n(\arccos x)$.

LeMma 1. For $-1<x<1$,

$$
\lim _{\rho \rightarrow 1^{+}} \sum_{m=0}^{\infty}\left(1-4 m^{2}\right)^{-1}\left(\rho^{2 m}+\rho^{-2 m}\right)^{-1} T_{2 m}(x)=(\pi / 8)\left(1-x^{2}\right)^{1 / 2} .
$$

\footnotetext{
* This lemma was proved by Dr. H. Dym.
} 
Proof. The sum above converges uniformly for any $\rho \geqq \rho^{*}>1$. Since

$$
\sum_{m=0}^{\infty}\left(1-4 m^{2}\right)^{-1} \cos 2 m \theta=\pi \sin \theta / 4,
$$

for $0<\theta<\pi\left[4\right.$, p. 39], and since $1<\rho^{2 m}+\rho^{-2 m}$, for $\rho>1$, it follows from Result 2 of Appendix A, that $\sum_{m=0}^{\infty \infty}\left(1-4 m^{2}\right)^{-1}\left(\rho^{2 m}+\rho^{-2 m}\right) T_{2 m}(x)$ is uniformly convergent for $\rho \geqq 1$, and that this sum equals $\pi\left(1-x^{2}\right)^{1 / 2} / 8$ when $\rho=1$.

Lemma 2. For $-1<x_{i}, x_{i}<1, x_{i} \neq x_{i}$,

$$
\lim _{\rho \rightarrow 1^{+}}(\rho-1 / \rho) \sum_{m=0}^{\infty}\left(\rho^{m}+\rho^{-m}\right)^{-1} T_{m}\left(x_{i}\right) T_{m}\left(x_{j}\right)=0 .
$$

Proof. Let $x_{i}=\cos \theta_{i}, x_{i}=\cos \theta_{i}, 0<\theta_{i}, \theta_{i}<\pi$, then the above sum has the form

$$
(\rho-1 / \rho) \sum_{m=0}^{\infty}\left(\rho^{m}+\rho^{-m}\right) \cos m \theta_{i} \cos m \theta_{i}=(\rho-1 / \rho) \sum_{m=0}^{\infty} b_{m}(\rho) a_{m}\left(\theta_{i}, \theta_{j}\right),
$$

where $b_{m}(\rho)=\left(\rho^{m}+\rho^{-m}\right)^{-1}$, and

$$
a_{m}\left(\theta_{i}, \theta_{i}\right)=\cos m \theta_{i} \cos m \theta_{i}=[\cos m \alpha+\cos m \beta] / 2 .
$$

with $\alpha=\left|\theta_{i}-\theta_{i}\right| \neq 0, \beta=\theta_{i}+\theta_{i}<\pi$.

Using identity (1) of Appendix A, we get:

$$
-\frac{1}{2}\left[\operatorname{cosec} \frac{\alpha}{2}+\operatorname{cosec} \frac{\beta}{2}\right] \leqq \sum_{m=0}^{\infty} a_{m} \leqq \frac{1}{2}\left[\operatorname{cosec} \frac{\alpha}{2}+\operatorname{cosec} \frac{\beta}{2}\right] .
$$

For $\rho>1,0<b_{m+1}<b_{m}$. Hence by Result 1 of Appendix A

$$
-b_{0} M<\sum_{m=0}^{\infty} b_{m}(\rho) a_{m}\left(\theta_{i}, \theta_{i}\right) \leqq b_{0} M,
$$

where $M=\frac{1}{2}[\operatorname{cosec} \alpha / 2+\operatorname{cosec} \beta / 2]$ and $b_{0}=\frac{1}{2}$. Now

$$
\sum_{m=0}^{\infty} b_{m}(\rho) a_{m}\left(\theta_{i}, \theta_{i}\right)=\left[\sum_{m=0}^{\infty} b_{m}(\rho) a_{m}\left(\theta_{i}, \theta_{i}\right)\right]-\frac{1}{4},
$$

and we have the following relation:

$$
\left|(\rho-1 / \rho) \sum_{m=0}^{\infty}\left(\rho^{m}+\rho^{-m}\right)^{-1} \cos m \theta_{i} \cos m \theta_{i}\right| \leqq(\rho-1 / \rho)\left(\frac{M}{2}+\frac{1}{4}\right),
$$

which yields the desired result.

Lemma 3. For $-1<x<1$,

$$
\lim _{\rho \rightarrow 1^{+}}\left\{(\rho-1 / \rho) \sum_{m=0}^{\infty}\left(\rho^{m}+\rho^{-m}\right)^{-1} T_{m}^{2}(x)-(\rho-1 / \rho) \sum_{m=0}^{\infty}\left[2\left(\rho^{m}+\rho^{-m}\right)\right]^{-1}\right\}=0 .
$$

Proof. For any $\rho>1$ the above left-hand side can be written as:

$$
\left(\frac{1}{2}\right)(\rho-1 / \rho) \sum_{m=1}^{\infty}\left(\rho^{m}+\rho^{-m}\right)\left(2 \cos ^{2} m \theta-1\right)=\frac{1}{2}(\rho-1 / \rho) \sum_{m=1}^{\infty} a_{m}(\theta) b_{m}(\rho),
$$


where $a_{m}(\theta)=2 \cos ^{2} m \theta-1$ and $b_{m}(\rho)=\left(\rho^{m}+\rho^{-m}\right)^{-1}$. Using the identity [4, p. 31]:

$$
\sum_{m=1}^{N} \cos ^{2} m \theta=\frac{N}{2}+\frac{\cos (N+1) \theta \sin N \theta}{2 \sin \theta},
$$

we get the following relation:

$$
\left|\sum_{m=1}^{N} a_{m}(\theta)\right| \leqq \operatorname{cosec} \theta .
$$

By Result 1 of Appendix A we have for any $\rho>1$ :

$$
\left|\sum_{m=1}^{\infty} a_{m}(\theta) b_{m}(\rho)\right| \leqq b_{1} M,
$$

where $b_{1}=(\rho+1 / \rho)^{-1}$ and $M=\operatorname{cosec} \theta$. Hence:

$$
\begin{aligned}
\mid(\rho-1 / \rho) \sum_{m=0}^{\infty}\left(\rho^{m}+\rho^{-m}\right)^{-1} T_{m}^{2}(x)-(\rho-1 / \rho) \sum_{m=0}^{\infty} & {\left[2\left(\rho^{m}+\rho^{-m}\right)\right]^{-1} \mid } \\
& \leqq M(\rho-1 / \rho)(\rho+1 / \rho)^{-1}
\end{aligned}
$$

and in the limit $\rho \rightarrow 1^{+}$, the left-hand side tends to zero.

LEMMA 4.

$$
\lim _{\rho \rightarrow 1^{+}}(\rho-1 / \rho) \sum_{m=0}^{\infty}\left(\rho^{m}+\rho^{-m}\right)^{-1}=\pi / 2 .
$$

Proof. By the substitution $\rho=e^{x}, x>0$, it remains to prove that

$$
\lim _{x \rightarrow 0} \frac{\sinh x}{x} \sum_{m=0}^{\infty} \frac{x}{\cosh m x}=\frac{\pi}{2} \text {. }
$$

Now:

$$
\frac{x}{\cosh (m+1) x} \leqq \int_{m x}^{(m+1) x} \frac{d y}{\cosh y} \leqq \frac{x}{\cosh m x},
$$

since $1 / \cosh y$ is monotonically decreasing for $y>0$. Hence:

$$
\int_{0}^{\infty} \frac{d y}{\cosh y} \leqq \sum_{m=0}^{\infty} \frac{x}{\cosh m x} \leqq \int_{0}^{\infty} \frac{d y}{\cosh y}+x,
$$

and in the limit $x \rightarrow 0^{+}\left(\rho \rightarrow 1^{+}\right)$we get:

$$
\lim _{x \rightarrow 0^{+}} \frac{\sinh x}{x} \sum_{m=0}^{\infty} \frac{x}{\cosh m x}=\int_{0}^{\infty} \frac{d y}{\cosh y}=\frac{\pi}{2} .
$$

An immediate consequence of Lemmas 3, 4 is:

Corollary 1. For $-1<x<1$

$$
\lim _{\rho \rightarrow 1^{+}}(\rho-1 / \rho) \sum_{m=0}^{\infty}\left(\rho^{m}+\rho^{-m}\right)^{-1} T_{m}^{2}(x)=\pi / 4 .
$$

The results, which are referred to in Section 5, are summarized below:

For $\rho \rightarrow 1^{+}$and $-1<x_{i}, x_{i}<1$ :

$$
\sum_{m=0}^{\infty}\left(1-4 m^{2}\right)^{-1}\left(\rho^{2 m}+\rho^{-2 m}\right)^{-1} T_{2 m}\left(x_{i}\right)=\pi\left(1-x_{i}^{2}\right)^{1 / 2} / 8+o(1) .
$$




$$
(\rho-1 / \rho) \sum_{m=0}^{\infty},\left(\rho^{m}+\rho^{-m}\right)^{-1} T_{m}\left(x_{i}\right) T_{m}\left(x_{i}\right)=\pi \delta_{i j} / 4+o(1)
$$

Department of Applied Mathematics

The Weizmann Institute of Science

Rehovot, Israel

1. R. E. BARNhILL, "Asymptotic properties of minimal norm and optimal quadrature," Numer. Math., v. 12, 1968, pp. 384-393. 28 \#393.

2. P. J. DAvis, Interpolation and Approximation, Blaisdell, Waltham, Mass., 1963. MR

3. R. Fletcher \& M. J. D. Powell, "A rapidly convergent descent method for minimization," Comput. J., v. 6, 1963/64, pp. 163-168. MR 27 \#2096.

4. I. S. GRADShTEYN \& I. M. RyzHIK, Tables of Integrals, Series, and Products, Fizmatgiz, Moscow, 1963; English transl., Academic Press, New York, 1965. MR 28 \#5198; MR 33 \#5952.

5. P. RABINOWITZ \& N. RICHTER, "New error coefficients for estimating quadrature error for analytic functions," Math. Comp., v. 24, 1970, pp. 561-570.

6. N. RICHTER, "Properties of minimal integration rules. II." (To appear.)

7. E. C. Trtchmarsh, The Theory of Functions, 2nd ed., Oxford Univ. Press, Oxford, 1939.

8. R. A. Valentin, Applications of Functional Analysis to Optimal Numerical Approximations for Analytic Functions, Ph.D. Thesis, Brown University, Providence, R.I., 1965. 